\title{
Christian Wevelsiep \\ »Vorpolitische Quellen oder vorpolitische Interessen?« \\ Politische Philosophie nach Heidegger und Arendt
}

\begin{abstract}
Einleitung
Diskussionen um die Begriffe Nation, Nationalismus und Patriotismus erleben eine unerwartete Konjunktur. Dies mag zum Teil auf politisch initiierte Werte- und Leitkulturdebatten zurück gehen, zum anderen Teil auf die unbeantwortete Frage nach der Möglichkeit gesellschaftlicher Integration und dies im besonderen im Horizont weltgesellschaftlicher Konfliktszenarien. Eine der vorrangigen Fragen ist dabei, ob die Homogenität eines Volkes zum Prozess der demokratischen Staatenbildung eine notwendige oder eher irreführende Voraussetzung ist, ob also eine relativ homogene soziale Basis als Grundlage der demokratischen Organisation des Staates zwingend vorauszusetzen ist oder ob sie vielmehr in nationalistischer Regression oder einem problematischen Volkssubstantialismus mündet. Derlei Insinuationen treten Demokratietheorien beherzt entgegen, indem sie die abstrakte Inklusion des Anderen in das rechtspositive Vokabular betonen und allein demokratische Praxis als strukturbildende Voraussetzung demokratischer Kulturen und eben nicht homogene vorpolitische Quellen anerkennen; politisch im Horizont zivilgesellschaftlicher Praxen, rechtsnormativ entlang der Ermöglichung volkssouveräner Verfahren.

Der vorliegende Aufsatz setzt an dieser Thematik an und möchte versuchen, den Begriff des Vorpolitischen als eine eigenständige Dimension des Politischen einzuführen.

Dabei geht es um die Frage, ob im Kontext vorpolitischer Sinnquellen nicht auch vorpolitische Interessen zu verorten wären, die nicht allein mit kulturellen Besonderheiten abzugleichen sind, da sie radikaler als etwa die Opposition von Fundamentalismus und Moderne das metapolitische Bedürfnis zum Ausdruck bringen, bestimmten Interessen zu folgen. Die vorpolitischen Quellen: gemeinsamer religiöser Hintergrund, gemeinsame Sprache, historische Bindungen, usw. münden zwar im günstigen Fall im Ideal eines Verfassungspatriotismus, wenn Gerechtigkeitsprinzipien in das dichtere Geflecht kultureller Wertorientierungen Eingang finden; Demokratietheoretiker mögen ferner postulieren, dass die Demokratie keine begründungstheoretische oder prozedurale Lücke aufweist, durch die eine vorpolitische Substanz eindringen könnte, bzw. dass eine Demokratie, die mehr als ein bloßer modus vivendi sein will, die vorpolitischen Quellen religiöser Lebensentwürfe und substanzieller Überzeugungen in demokratische Prozesse einzubinden vermag. Um aber die Wucht gesellschaftlicher Konflikte plausibel zu ma-
\end{abstract}


chen, muss möglicherweise ein weiteres Begriffselement angenommen werden, das zwischen dem Dualismus politischer und vorpolitischer Wir-Gemeinschaft zu verorten ist.

Im einzelnen soll der Begriff des Vorpolitischen entlang klassischer politischer Entwürfe konturiert werden; zunächst in Opposition zum Horizont einer vorpolitischen Homogenität bei Heidegger und Schmitt (1); anschließend in einer ausführlichen Auseinandersetzung mit der politischen Theorie Hanna Arendt's (2). Deren politischer Freiheitsbegriff ist einerseits maßgeblich für die Explikation des vorpolitischen Sinnhorizonts; zugleich eröffnet er offene Flanken, von denen hier vor allem die rigide Unterscheidung von politischen und vorpolitischen Interessen $\mathrm{zu}$ nennen ist (3). Das hieran anschließende Schema von vorpolitischem Fundamentalismus und politischer Moderne bringt in diesem Zusammenhang die Schwierigkeit der politischen Theorie zum Ausdruck, vorpolitische Interessen als eigenständige Dimension des Politischen anzuerkennen.

\section{Zur philosophischen Explikation der vorpolitischen Homogenität bei Heidegger und Schmitt}

Dass die Einheitlichkeit eines Volkes zu den notwendigen vorpolitischen Voraussetzungen zur Bildung eines Staates zähle, ist eine Annahme von schwerwiegender Tradition. Die klassische demokratische Staatstheorie setzte auf eine soziale Basis freier und gleicher Bürger, die entlang angenäherter sozialer Gleichheit die Bildung eines einheitlichen Willens, die friedliche Austragung von Konflikten und die Angleichung der Interessen ermöglichte. ${ }^{1}$ Jenseits der Frage, ob die klassische Staatstheorie diese soziale Basis einforderte oder aber als gegebene Voraussetzung voranstellte, lassen sich von dieser Position aus mehrere, für die Politikwissenschaft relevante Unterscheidungen ableiten: in einer Hegelianischen Linie, bei der Nation, Nationalbewusstsein und Patriotismus dem Staat folgen und ihm insofern nicht vorangehen; ferner in vergleichbaren wirkmächtigen Geschichtsschreibungen, die davon ausgehen, dass Nationen durch staatliche Macht konstruiert und über Schule, Militär, Amtssprache, usf. gebildet werden. ${ }^{2}$

Die Dekonstruktion dieser wirkmächtigen Konstruktionen der Nation ist seit geraumer Zeit etabliert; ${ }^{3}$ sie sieht in ihr - pauschal gesprochen - gelingende Projekte, hegemoniefähige Konstruktionen und hegemoniale symbolische Ordnungen zu verankern, die einerseits an reale Erscheinungen wie Sprache, Religion, Tradition anknüpfen, zugleich aber entlang vermeintlich geschichtsverklärender Positionen hieraus die Grundlage einer gemeinsamen identitätsstiftenden Kultur ableiten.

Der Umweg über Heidegger und Schmitt hat zunächst eine negative Funktion, denn er dient der Abgrenzung; schließlich gilt es zu zeigen, inwiefern der Begriff des Politischen durch den zum Teil opaken Begriff des Vorpolitischen in normative Sackgassen führt. Das Vorpolitische hat - wie im folgenden noch zu zeigen ist - eine aktuelle Di-

1 Franz Neumann, Die Herrschaft des Gesetzes, Frankfurt 1980.

2 Bernd Giesen, Die Intellektuellen und die Nation, Frankfurt a. M. 1993.

3 Benedict Anderson, Die Erfindung der Nation, Frankfurt / New York 1996.

ZfP 56. Jg. 3/2009 
mension, denn es kreist um die Frage, ob und wie sich eine politische Ethik auf dem Boden der Gemeinschaft gründen ließe, bzw. ob die Gemeinschaft aus den Quellen der modernen Subjektivität und deren radikaler Subjektivität heraus verstanden werden solle. Was immer Heidegger politisch verschlüsselt haben mag - die Ablehnung des Universalismus, die Betonung der Einzigartigkeit geschichtlicher Konstellationen und die Immanenz je besonderen Leidens verweisen auf die politischen brisanten Fluchtlinien der Heideggerschen Philosophie.

Es ist vor allem Heideggers geschichtliches Denken, das für ihn die ganze Fragwürdigkeit der Metaphysik aufwirft. ${ }^{4}$ Das metaphysische Denken richtete sich demnach in der Unwandelbarkeit letzter Sinnbezüge ein, wohingegen eine radikal gefasste Idee von Geschichtlichkeit jeden universalistischen Geltungsanspruch zerstörte. Dass geschichtliches Leben zur Grundlage des Philosophierens werde, bedeutet, dass das menschliche Leben sich in seiner Eigentlichkeit zeigen kann. Eigentlichkeit und Vergegenständlichung stellen den wegweisenden Kontrast dar, an dem sich das menschliche Leben zu orientieren vermag. Letzteres entgleitet uns, wenn es sich aufs Objektive und Gegenständliche versteift, wenn im objektivierenden Bezug das Erleben der Lebenswelt, die uns begegnende Welt entweltet wird. Heideggers gelebter Augenblick avisiert die Selbstdurchsichtigkeit der Lebensvollzüge, er wird zur Kunst des Wachseins des Daseins für sich selbst.

Da sich die Daseinsweisen nicht alle gleich gut entsprechen, entsteht ein hierarchisches Gefälle; mit dem Eigenen als dem Ursprung, dem Eigentlichen und dem Wesen erhält man einen normativ sich geltend machenden Maßstab. Die Ereignishaftigkeit des Daseins ist in diesem Sinne bereits die höchste Selbstdurchsichtigkeit, die letzteres für sich selbst erreichen kann. Selbstverdeckung als Uneigentlichkeit, Selbsttransparenz als Eigentlichkeit - mit dieser doppelten Gleichung wird ein Abgrund der Freiheit eröffnet.

An just dieser Stelle wird aber auch ein politisch-ethischer Bruch deutlich, der die vermeintlich normative Neutralität der Eigentlichkeitssemantik in nicht unproblematische Gefilde überführt. Heidegger bringt seine Ethik der Eigentlichkeit gegen eine Ethik der Öffentlichkeit in Stellung. Er setzt sich von der aristotelischen Tradition einer praktischen Ethik des öffentlichen Lebens ab: Aristoteles hatte bekanntlich die »Philosophie des Guten « auf den Boden der gesellschaftlichen Wirklichkeit seiner Zeit gebracht, er hatte das sittlich Gute nicht in Abgrenzung zum gesellschaftlich Geltenden, sondern im Anschluss an es gewonnen. Mit dieser weitreichenden Tradition erhält man eine Orientierungsgröße für gelingendes und ethisch verantwortliches Leben, das Heidegger diffamierend die Welt des »Man« bezeichnet. Das Selbst des »Man « wird bei Heidegger aus eben dieser uneigentlichen Welt des »Man « zurückgeholt, um es zu dem Bewusstsein der Sterblichkeit und der Zeit zu bewegen, aber auch, um ihm die Einsicht in die Unverlässlichkeit aller zivilisatorischen Daseinsfürsorge zu verschaffen. ${ }^{5}$ Das bei sich selbst ankommende Dasein ist bodenlos und losgerissen; es erhält keinen anderen Halt als das

4 Martin Heidegger, Die Technik und die Kebre, Pfullingen 1962; Ders, Sein und Zeit, Tübingen 1963.

5 Heidegger, Sein und Zeit, a.a.O., S. 310. 
Bewusstsein des eigenen Seinkönnens. Aus diesem existentiellen Abgrund erst entsteht die menschliche Größe im Sinne von Spontaneität und Schöpfertum.

Es geht dann nicht primär um das gute und ethisch richtige Handeln, sondern vorrangig um die Intensitätssteigerungen des Daseins; um die Öffnung für große Augenblicke, mithin um die Entschlossenheit zum eigentlichen Leben. Gleichwohl betont Heidegger, dass Philosophie weder moralische Auskunftsinstanz sei, noch Anweisung und Orientierung verschaffen würde. Es geht um nichts mehr und nichts weniger als das Abtragen von vermeintlich ethischen Objektivitäten, die ein Nichts hervortreten lassen - freilich ein Nichts, das zu seinem eigenen Seinkönnen auffordert.

Für Heidegger war bekanntermaßen ein authentisches Leben, das der praktischen Frage nach dem eigenen Sein nicht ausweicht, nicht möglich im Medium des öffentlichen Lebens einer demokratischen Bürgergesellschaft; um den Solipsismus der isolierten Existenz auszugleichen, musste er einen Ersatz fingieren und fand ihn im "geschichtlichen Volk $\ll$.

Dabei vermengt er aber diese eigentlich existentialistische Aufforderung zur Stellungnahme mit einem problematischen Geschichts- und Politikverständnis: der generalisierte Andere verschwindet zusammen mit dem entfremdeten »Man« der manipulierten Öffentlichkeit, an seine Stelle tritt der »stille Ruf des Gewissens « und die Verabsolutierung des »geschichtlichen Volks ${ }^{6}{ }^{6}$

In dieser geschichtsphilosophischen Fluchtlinie, die verkürzt gesagt die Betrachtung des Volkes nur in Kategorien von Schicksals- und Willensgemeinschaft fassen kann, ist dann auch: Carl Schmitts Postulat der nationalen Homogenität als Voraussetzung der Demokratie zu verorten. Ohne allzu große Vereinfachung lässt sich Schmitts Politikbegriff als ein Versuch beschreiben, das Politische als die gegenüber dem Staat und den Institutionen fundamentalere Dimension zur Geltung zu bringen. Das Politische wird hier zu einer vorstaatlichen Kategorie, zu einem Intensitätsgrad einer Verbindung, resp. Trennung, die zugleich eine ordnungsstiftende Entscheidung in sich trägt. Verbindung und Trennung gehören zusammen, insoweit erst beide die Unterscheidung von innen und außen bedingen und damit erst die Möglichkeit einer substantiellen und handlungsfähigen Einheit. Nicht die Tatsache von Assoziation und Dissoziation ist dabei aber entscheidend, sondern vielmehr deren Intensität. Deren äußerer Grad erhält seine produktive Spannung aus der Unterscheidung von Freund und Feind. ${ }^{7}$

Während der Rechtspositivismus bekanntermaßen den Grund der Geltung des Rechts in rechtlichen Normen findet, so erkennt der dezisionistische Rechtsbegriff dagegen die außerrechtliche und herrschaftssoziologische Basis aller Rechtsgeltung an.

Die Entscheidung entspringt mit Schmitt einem normativen Nichts; sie ist etwas völlig anderes als das Resultat einer Rechtsbegründung, denn sie wird nicht mit Hilfe einer Norm zugerechnet, sondern sie bestimmt vielmehr den Zurechnungspunkt, von dem aus Norm und normative Richtigkeit rekonstruierbar werden. ${ }^{8}$ Rechtsnormen lassen sich

6 A.a.O. S. 77.

7 Carl Schmitt, Der Begriff des Politischen, München 1932, S. $26 \mathrm{ff}$.

8 A.a.O., S. 42.

ZfP 56. Jg. 3/2009 
nicht restlos aus höherem Recht und Gerechtigkeitsbestimmungen ableiten, sondern es ist die Entscheidung in ihrem nicht weiter begründungsnotwendigen Selbstbezug, die der Normbegründung ihre Rechtskraft verleiht. Die Basis der Rechtsgeltung ist von daher als knapp zu beschreiben, als sie gewissermaßen einem nicht bestimmbaren und nicht verfügbaren Augenblick des Entscheidungsgebers entspringt.

Diesem knappen Moment der Nicht-Verfügbarkeit des Rechts entspricht eine ambivalente Ineinssetzung von Souveränität und Herrschaft. Das Grundproblem der Rechtstheorie entspringt demgemäß nicht der inhaltlichen Normativität des Rechtsgebots, sondern der formalen Frage der letzten Entscheidungsinstanz. Die Rechtsnorm mag zwar festlegen, wer entscheidet und wer interpretiert; das für Schmitt wesentliche Problem verschiebt sich aber ins Unendliche, solange nicht die Frage nach dem letztentscheidenden politischen Willen gestellt wird. ${ }^{9}$

Für Schmitt ist konsequenterweise ein solches Verfahren nicht gangbar, das nach der Legitimität der politischen Einheit des Staates im Sinne einer inhaltlichen Rechtfertigung fragt. Die herrschaftssoziologische Intransingenz des Dezisionismus führt somit zu einer folgenreichen Dekonstruktion des Legitimitätsgedankens: Ein Staat, der die politische Einheit eines Volkes zum Ausdruck bringe, existiere allein der Sphäre des Politischen und sei ferner einer Rechtfertigung weder bedürftig noch fähig; er agiere vielmehr in einem Beziehungs- und Spannungsfeld, in dem noch die Anwendung physischer Gewalt auf das Recht auf Selbsterhaltung zurückgehe: Freund und Feind, Eigenes und Fremdes versuchen sich in ihrer jeweiligen Besonderheit zu erhalten, so dass nicht Legitimität im Sinne der individuellen Freiheit die innere Rationalität des Geltungsgrundes des Staates markiere. Um dem Bestandssicherungsinteresse gerecht zu werden, bestimmt sich die politische Herrschaftseinheit in einer Entscheidung immer selbst und muss dabei nach Schmitt ihr inneres Konfliktfeld unterhalb der Freund-Feind-Schwelle halten.

Im Fluchtpunkt des von Schmitt und anderen verfolgten Demokratieverständnisses freilich wartet der »lange Schatten des Staatswillenspositivismus «. ${ }^{10}$ Beispielhaft am Weimarer Verfassungsdiskurs lässt sich eine Form der Staatsrechtslehre verfolgen, in der der Staat als von außen »impermeable Rechtsperson «11 selbständig über seine inneren Angelegenheiten befindet. Ein solchermaßen verselbständigter Staat stellte der damaligen Auffassung nach eine Ordnung eigenen Rechts dar, die sich mit der rechtsstaatlichen und parlamentarischen Verfassung der privaten Angelegenheiten der Gesellschaft »allenfalls berühren, niemals aber überschneiden durfte. «. ${ }^{12}$ Dieser Staat ist als Besitzer der Rechts-

9 Carl Schmitt, Politische Theologie. Vier Kapitel zur Lebre von der Souveränität, Berlin 1934 (1922), S. 46.

10 Hauke Brunkhorst, Politik der Menschenrechte. Zur Verfassung der Weltgesellschaft.

In: Armin Nassehi/Markus Schroer, (Hg.), Der Begriff des Politischen, Baden-Baden 2003, S. 71-89;

Ders., Der lange Schatten des Staatswillenspositivismus. Parlamentarismus zwischen Untertanenrepräsentation und Volkssouveränität. In: Leviathan 3 (31), 2003a, S. 362-382.

11 Brunkhorst, Der lange Schatten, a.a.O., S. 356.

12 Ebd. 
ordnung jederzeit in der Lage, sich von seinem »Besitz « zu trennen und sich auf seine überpositiv legitimierte Herrschaftsmacht zu berufen.

Die Ausführungen machen deutlich, dass mit dem Postulat der nationalen Homogenität als Voraussetzung der Demokratie eine normativ brisante Drift einhergeht: das nationale Wir-Gefühl, das für den Kitt des gesellschaftlichen Zusammenhalts zu sorgen hat, ist dann mit dem Preis verbunden, sich dem Fremden, Heterogenen und Andern abzugrenzen.

Homogene und geschichtsphilosophisch verbrämte vorpolitische Voraussetzungen können insofern in rigiden staatswillenspositivistischen oder kollektivistischen Unterscheidungen münden; vereinfacht gesprochen: das nationale Wir-Gefühl, das für den Kitt des gesellschaftlichen Zusammenhalts zu sorgen hat, ist dann mit dem Preis verbunden, sich dem Fremden, Heterogenen und Andern abzugrenzen, während Herrschaft und Souveränität ineins gesetzt werden. Ohne direkt in bellizistischen oder militärischen Engführungen zu münden, zeigt Schmitts Begriff des Politischen eine starke Affinität zum existentiellen Kampf zwischen Leben und Tod; das Feind-Freund-Schema lässt sich als unmittelbar wirklichkeitsbezogene Kategorie aus nichts Äußerem ableiten als der Schwere der realen Konfrontation. Als »Letztunterscheidung « verweist die FreundFeind-Distinktion nicht nur auf die Tautologie eines unbestimmten Fremden, sondern zugleich auf einen totalitären Begriff politischer Wirklichkeitserschließung. An den äußersten identitätslogischen Rand getrieben, kann er nicht »empirisch « bestritten werden, wohl aber dessen emphatische Begrüßung hinterfragt werden.

Es ist somit wohl nicht zuletzt Schmitts offenkundige Geringschätzung, mit der er normative Ansprüche ethischer Art als fiktive Normativismen degradiert, die den Dezisionismus in ein offen antiliberales Klima versetzen. Der Kampfcharakter des Politischen ist nicht nur jenseits der wirtschaftlichen Konkurrenz oder der geistigen Diskussion $\mathrm{zu}$ verorten; die Vorstellung einer durchgehend pazifizierten Welt wird dort dekonstruiert, wo sie auf eine opfer- und leidfreie Welt, auf eine kryptopolitische Leere zusteuert. Für Schmitt ist das Politische somit eine Chiffre einer anti-aufklärerischen Staatsethik, die der geschichtlichen Existenz Leben und Sinn verleiht (eine mit Blick auf die aufbrandenden Kulturkämpfe der Moderne höchst aktuelle Deutung); der politische Existentialismus, der hieraus resultiert, lässt den modernen Menschen sein individuelles Sekuritätsstreben überwinden und in der Bereitschaft zum Opfer, resp. im Phantasma einer überindividuellen Verbindlichkeit aufgehen.

Die Vergleichgültigung des Subjekts ist es nicht zuletzt, die den politischen Existentialismus in fundamentalistische Sackgassen laufen lässt, wobei Schmitt letztlich den Beweis schuldig bleibt, warum die Freund-Feind-Unterscheidung in einem ontologischen Antagonismus münden muss.

\section{Zum Begriff vorpolitischer Freibeit bei Arendt}

Im Horizont des Jus Publicum Europaeum sucht Schmitt nach den tiefer gelegten Fundamenten des Politischen und findet sie im geschichtlichen Ursprung des revolutionären 
Ausnahmezustands. Dem Verfassungsgesetz liegt die geschichtliche Substanz der existentiellen Entscheidung eines höheren Subjekts voraus und diese legt die Differenz zwischen konkreter und abstrakter Ordnung fest. Schmitt identifiziert hierfür zwei Momente, die für die Völkerrechtsverfassung seit dem Westfälischen Frieden bestimmend sein sollen: die umfassende europäische Raumordnung und die durchgängige, anerkannte Wirtschaftsverfassung des liberalen Konstitutionalismus.

Die These entstaatlichter Großräume, die von Hegemoniemächten dominiert werden und der Abwehr raumfremder Mächte verpflichtet war, wandelte sich in eine Synthese aus europäischer Staatenegalität und globalem Imperialismus. Zwischen Europa und der übrigen Welt konnte es dementsprechend keine Gleichheit geben, insofern es bei den wilden Völkern keine Zivilisation, kein öffentliches Recht und keine Staatlichkeit gebe, nur der starke Arm des Hegemons kann hier konkrete Ordnung durch ein elastisches Maßnahmenrecht schaffen.

Man könnte es sich an dieser Stelle einfach machen und auf die politiktheoretischen Alternativen verweisen, etwa auf die von Wingert ${ }^{13}$ konturierte Unterscheidung negativer oder negativistischer Bezugspunkte demokratischer Gesellschaften oder auch auf die von Nancy Fraser oder Hauke Brunkhorst erhoffte starke Weltöffentlichkeit im Kommen. Im folgenden soll aber ein anderer, sicherlich widerspruchvollerer Weg beschritten werden, der sich auf die Umrisse des vorpolitischen Interessebegriffs stützt und eine soziologische Alternative zu den oben beschriebenen Verengungen kennzeichnet.

Der Begriff des Vorpolitischen hat, wie gesehen, eine lange Tradition und zugleich einen langen Schatten. Er verweist auf diejenige Sphäre, die vor jeder politischen $\mathrm{Zu}-$ sammenkunft ein basales soziales Geschehen im vorrechtlichen und vorpolitischen Bereich ermöglicht und gewissermaßen vorbereitet; vereinfacht gesprochen: es ist dasjenige Selbstverhältnis, von dem aus politische Interessen überhaupt erst möglich werden.

Das vorpolitische Interesse bleibt zwar ein Begriff im Zwiespalt, denn seine Latenz und Nichtsichtbarkeit speist sich aus einem existentialistischen Verhältnis, eines Geworfenseins (Heidegger), das vor jeder politischen Festlegung Freiheit ermöglicht und bedingt.

Insoweit können wir Heidegger also folgen. Es hat aber - wie im folgenden zu zeigen ist - durchaus politische Relevanz. Das Vorpolitische ist nicht das Unpolitische, es ist also nicht mit einem rein immanenten Gemeinschaftsbegriff abzugleichen.

Hannah Arendt markierte in ihrer politischen Theorie einen wesentlichen Unterschied, indem sie die Möglichkeit eines authentischen Lebens an sein öffentliches Erscheinen bindet. Nur im Lichte der Öffentlichkeit kann sich wahres oder eigentliches Leben zeigen. An die Stelle der elitären Verabsolutierung des geschichtlichen Volkes treten intersubjektive Begriff der Macht, der Pluralität und des welterschließenden Handelns. Aus der Fülle ihrer politischen Ansätze und Theoreme ist im folgenden den Begriff

13 Lutz Wingert, Unpathetisches Ideal. Über den Begriff eines bürgerschaftlichen Wir, In: Hauke Brunkhorst, (Hg.), Demokratischer Experimentalismus, Frankfurt a. M. 1998, S. 33-44. 
der Handlung exemplarisch herauszugreifen, denn an ihm zeigt sich, wie das Vorpolitische gegen Heidegger und Schmitt zu verteidigen ist.

Zunächst einmal ist die Abtrennung des Arendt'schen Handlungsbegriffs vom Telos des Guten in den Mittelpunkt zu rücken. ${ }^{14}$

Arendt's Idee politischer Freiheit ist in diesem Sinne radikal zu nennen, insofern sie auf einen Bruch mit dem Kontinuum der Geschichte zielt. Der Grundgedanke, der hier aufgegriffen wird, erkennt kein umfassendes Gesetz mit unbedingter Geltung an, sondern lediglich die unbegrenzte Wiederholbarkeit einer gerechten und befreienden Praxis.

Dies bedeutet: das konkrete Emanzipationsgeschehen ist jederzeit und überall wiederholbar und zugleich immer wieder anders; der konkrete Kampf der Menschen um Freiheit, Würde und Gerechtigkeit entzieht sich der Logik des allgemeinen Gesetzes. Arendt wendet sich gegen die teleologische, resp. universalistische Hypothek, die das Politische in letzten Gewissheiten und einer alle besonderen Fälle übergreifenden Gesetzgebung münden lässt.

Diese Dimension stellt explizit den Fundierungszusammenhang zwischen den politischen Freiheitsimpulsen der Moderne und dem Menschenrechtsuniversalismus der liberalen Tradition ins Zwielicht und umschreibt ihn als radikalen Bruch mit dem Kontinuum der Geschichte, als radikalen Neubeginn. Die sich dabei abzeichnende Endlichkeitsphilosophie interpretiert die Konstitution eines Raums öffentlicher Freiheit als kontingenten performativen Akt und dies ist eben jener bedeutende Ort des Unbestimmten, von dem aus $\mathrm{H}$. Arendt einen Anti-Universalismus entwickelt, der zwar zu profunden Aussagen über die politische Freiheit republikanischer Staatsformen führt, der aber ebenso den grundlegenden Zusammenhang zwischen der Idee politischer Freiheit und dem Universalismus des Menschenrechts zerteilt, ohne ihn zu zerstören. Das entscheidende Moment ihres politischen Denkens findet sich in der Bestimmung eigentlichen Handelns als Neuschöpfung aus dem Nichts. Freiheit ist für sie die vorpolitische Quelle politischen Handelns und sie besteht vor allem darin, dass verschiedene Akteure zu einer gemeinsamen Sache Stellung nehmen können. Die gemeinsame Sache stiftet eine gemeinsame Welt und die vorbürgerliche Elementarrepublik bildet sich über all dort, wo Gründe im ständigen Wechsel der Perspektiven ausgetauscht und gewogen werden. Der Ort des Interesses an der Sache selbst ist dann ein kommunikativer Zwischenraum, der als Urform des öffentlichen Raumes fungiert und von dem aus Arendt eine durchaus einseitige Analyse der modernen Gesellschaft entwirft, die dann gesondert zu kritisieren wäre, wie sicherlich auch die zwiespältige Haltung zum Nationalstaat oder zur Rolle positivierter Menschenrechte.

Konzentriert man sich aber auf den Aspekt des vorpolitischen Handlungsbegriffs, dann fällt die rigide Unterscheidung zwischen politischen und vorpolitischen Lebewesen in den Blick.

14 Hannah Arendt, Elemente und Ursprünge totaler Herrschaft. Frankfurt 1955; Ders., Vita activa oder: vom tätigen Leben, Stuttgart 1960; Ders., Über die Revolution. München 1974; Ders., Macht und Gewalt. München 1975; Ders.: Zwischen Vergangenheit und Zukunft, München 1994.

ZfP 56. Jg. $3 / 2009$ 
Für Arendt gab es bekanntermaßen nur ein einziges Menschenrecht: Das Recht, Rechte zu haben, war in ihren Augen das grundlegende Recht, einer politisch organisierten Gemeinschaft anzugehören und in einem politischen Bezugssystem zu leben. Ein höherstufiges Recht auf Rechte wird zum vorstaatlichen Weltbürgerrecht erklärt, das eben nichts anderes als eine geschichtliche Möglichkeit des gegenwärtigen Zeitalters zum Ausdruck bringt; Menschsein heißt dann für sie: an öffentlichen Angelegenheiten Teil zu nehmen, der Mensch ist von daher politisches Wesen, das sich von allen unpolitischen Lebewesen unterscheidet. ${ }^{15}$

Die Schwäche dieser Argumentation liegt auf der Hand: denn ein solches Recht kann zunächst nicht mehr beinhalten, als dass es einer politischen Gemeinschaft selbst überlassen bleibt, sich selbst Recht zu geben und zu verschaffen. Was für Rechte das wären und ob sie egalitär wären, bliebe in dieser Logik der Gemeinschaft selbst überlassen. Aber die Argumentation von Arendt hat noch eine weitere Problematik, denn nur der freie Bürger hat nach Arendt die volle Würde, Rechte zu haben und diese klassische aristotelische Unterscheidung wird noch einmal verschärft zur Dichotomie zwischen politischen und unpolitischen Lebewesen. An diesem Punkt wird es notwendig, über Arendt hinaus zu denken und die Rigidität der Unterscheidung zwischen dem politischen und vorpolitischen Handlungsbegriff aufzubrechen, indem der Begriff des vorpolitischen Interesses Arendts politischer Anthropologie entgegen gehalten wird.

\section{Zur Differenz politischer und vorpolitischer Interessen}

Theorien, die den ursprünglichen Konfliktcharakter der Moderne auffangen wollen, müssen den agonalen Charakter des Sozialen, Auseinandersetzungen zwischen Zentren und Peripherien, Klassen und Bewegungen und Hegemonien verorten, sie müssen ferner den potentiell einheitssprengenden Konfliktcharakter entlang der Artikulation politischer Interessen begrifflich öffnen.

Eine Analytik des Vorpolitischen betreibt in diesem Sinne keine bloße Ergänzung dieses Vokabulars, sondern sie zielt auf eine Art Umstülpung des liberalen Bildes der Politik ab, indem sie jene Strukturen des Politischen sichtbar machen will, die in der liberalen Selbstbeschreibung als selbstverständlich vorausgesetzt oder marginalisiert werden. Es geht in dieser Perspektive um implizite symbolische Ordnungen, die spezifische kulturelle Codes bereitstellen, die sich dann in Diskursen und sozialen Praktiken ausdrücken; dort, wo z. B. die liberale Selbstbeschreibung danach fragt, wie politische Herrschaft ihre Legitimität als Ausdruck gesellschaftlicher Interessen erreichen kann, wird hier die Frage aufgeworfen, welche stillschweigend vorausgesetzten Codierungen definieren, was diese Interessen und Ziele überhaupt sind, also nach dem, was die unbewussten, unsichtbaren, eben: vorpolitischen Bedingungen des Politischen sind. Es »gibt«

15 Arendt, Über die Revolution, a.a.O., S. 75. 
in diesem Sinne elementare soziale Prozesse, nach denen sich die soziale Ordnung schon immer selbst erschafft, bevor Ordnungsvorhaben auf den Plan treten. ${ }^{16}$

Der Vorschlag lautet: den Begriff des vorpolitischen Interesses in die politologische Arithmetik aufzunehmen, denn dieser Begriff vermeidet eine Engführung über rein politische Interessen, die den systemisch-funktionalistischen Deutungen zu eigen sind, als auch den abstrakten Verweis auf vorpolitische Räume oder Quellen. Ein möglicherweise irreführender Dualismus ergibt sich, wenn man davon ausgeht, dass die vorpolitischen Quellen: gemeinsamer religiöser Hintergrund, gemeinsame Sprache, historische Bindungen, usw.im günstigen Fall im Ideal eines Verfassungspatriotismus münden.

Man müsste das differenzieren: normativ betrachtet ist es richtig, wenn Gerechtigkeitsprinzipien in das dichtere Geflecht kultureller Wertorientierungen Eingang finden; Demokratietheoretiker mögen ferner postulieren, dass die Demokratie keine begründungstheoretische oder prozedurale Lücke aufweist, durch die eine vorpolitische Substanz eindringen könnte, bzw. dass eine Demokratie, die mehr als ein bloßer modus vivendi sein will, die vorpolitischen Quellen religiöser Lebensentwürfe und substanzieller Überzeugungen in demokratische Prozesse einzubinden vermag.

Um aber die Wucht gesellschaftlicher Konflikte plausibel zu machen, muss möglicherweise ein weiteres Begriffselement angenommen werden, das zwischen dem Dualismus politischer und vorpolitischer Wir-Gemeinschaft zu verorten ist. Die politikwissenschaftliche Tradition zieht bekanntermaßen eine scharfe Trennlinie zwischen rationalistisch-analytischen Perspektiven und solchen, die vorpolitische Sinnhorizonte zum Maßstab politischer Theorie erheben. Von der Analyse gouvernmentaler und hegemonial-universaler Strukturen zur normativen Explikation demokratisch verfasster Gesellschaften ist es dann ein weiter, durch kategoriale und erkenntnistheoretische Welten getrennter Weg, der hier auch nicht vorschnell zusammen zu fügen ist.

Gegenüber einer Vorrangstellung oder Negierung vorpolitischer Räume, Quellen oder Sinnhorizonte soll im vorliegenden Fall diskreter von vorpolitischen Interessen zu sprechen sein. Die Begrifflichkeit vorpolitischer Interessen verhindert zunächst, dass sich der Anspruch politischer Integration vorschnell auf das Gelingen, bzw. das Vorhandensein demokratischer Kultur reduziert. Eine solche Öffnung der konflikttheoretischen Soziologie ist dort vonnöten, wo die Evolution der Weltgesellschaft nicht der emergenten Logik eines neuen Gesellschaftsmodells folgt, sondern sie begrifflich fassbar als Vervielfältigung von Akteuren, Interaktionsebenen, Herrschaftsverhältnissen und Logiken kollektiven Handelns wird. Die widerstreitenden Faktoren der sozialen Integration und Desintegration sind dann zwar in einschlägigen konfliktsoziologischen Mehrebenensemantiken aufzufangen und zu integrieren; anthropologische Dimensionen sind aber darüber hinaus dort zu betonen, um zu einer eigenständigen politischen Theorie zu gelangen,

16 Andreas Reckwitz, Die Politik der Moderne aus kulturtheoretischer Perspektive: Vorpolitische Sinnhorizonte, symbolische Antagonismen und das Regime der Gouvernmentalität, In: Birgit Schwelling, (Hg.), Politikwissenschaft als Kulturwissenschaft. Theorien, Methoden, Problemstellungen, Wiesbaden 2004, S. 33-57. 
die im wesentlichen erfahrungskonstituiert ist. ${ }^{17}$ Geht man davon aus, dass es Basisdispositionen individuellen Handelns gibt, welche die Grenzen freier Politik und des Rechts bilden, stößt man zumeist auf Bedürfniskollektionen, die Aspekte wie Selbsterhaltung, Tätigkeit, Erlebnis und Genuss, aber auch: Freiheit und Schaffen, Erwerb und Besitz, Soziabilität und Gesellung nennen ${ }^{18}$. Über die vitalen Lebensinteressen im sozialen Verband hinaus lassen sich solche Interessen- und Bedarfslisten auf die Grundbedürfnisse personal-sozialer Entfaltung sowie der Öffnung zur Metaphysik und existentieller Interpretation und Integration zusammen fassen. Die Frage nach den vorpolitischen Interessen fügt sich in diese Reihe, ohne sich zugleich einer Grunddisposition zwingend zuordnen zu lassen; sie oszilliert quasi zwischen den Werten der Selbsterhaltung und der Verwirklichung des Eigenwertes. Anthropologisches Denken, das die o. g. Bedürfnisse in Richtung individueller Lebenspläne entwirft, behauptet, dass es diesen Dispositionen vorhergehende Interessen gibt, die als vorpolitische ein metapolitisches Bedürfnis Ausdruck bringen: das grundlegende Interesse, im eigenen Interesse zu handeln.

Politische Interessen ermöglichen bekanntermaßen politische Beteiligung und bürgerschaftliches Engagement, dieses ist bis zu einem bestimmten Grade messbar und lässt auch Rückschlüsse auf Ausmaß, Qualität und Entwicklung politischer Partizipation zu. Vorpolitische Interessen hingegen bringen die Bedingungen einer subjektiven Praxis zum Ausdruck, die rein rationalistischen Akteursperspektiven entgehen. Vorpolitische Interessen umschreiben das nicht restlos logofizierbare Interesse, ein bestimmtes Interesse zu haben und zu verfolgen. Während das Subjekt in funktionalistischen oder normativonotologischen politischen Theorien »nur eine Projektionsfläche passiver Träger der Nomothetik einer universalen Vernunft « bleibt, ${ }^{19}$ setzt eine wesentlich erfahrungskonstituierte und an der Logik des Unbestimmten orientierte Theorie an einem Subjektbegriff an, dessen Handlungsbestimmungen sich nicht restlos logofizieren lassen und deren Praxis weder den logischen Postulaten einer Friedenswissenschaft noch einem »prädeterminierten Aktualisierungsplan « folgt. ${ }^{20}$ Ohne in die Niederungen politischer Romantik abzugleiten, zeichnet sich ein solcher Ansatz dadurch aus, das das hier zugrunde liegende Politische nicht determiniert ist und wesentlich darin besteht, einen Raum politischer Freiheit zu eröffnen.

Wiederholt zu betonen ist: die Unterscheidung von politischen und vorpolitischen Interessen ist keine exklusive, sie ist nicht an der Logik der gewaltsamen Ausschließung interessiert und versucht vielmehr, ihre Geltung aus dem Ineinander politischer und vorpolitischer Interessen zu gewinnen.

Versteht man Interesse im psychologischen Sinne als kognitive Anteilnahme oder Aufmerksamkeit, die eine Person an einer Sache oder einer anderen Person hat, dann liessen sich hieran anschließend Interesse und Desinteresse, situative Interessen bis hin

17 Klaus Gerd Lutterbeck, Apriorische Politikwissenschaft, In: Zeitschrift für Politikwissenschaft (54), 1, 2007, S. 21-40; ferner: Wilfried Brugger, Das anthropologische Kreuz der Entscheidung in Politik und Recht, In: Zeitschrift für Politikwissenschaft 3, 2005, S. 261-273.

18 Brugger, Das anthropologische Kreuz, a.a.O., S. $261 \mathrm{ff}$.

19 Lutterbeck, Apriorische Politikwissenschaft, a.a.O., S. 33.

20 A.a.O., S. 34. 
zu rechtlichen und Vertragsinteressen unterscheiden. Politisch artikulierbar werden Interessen darüber hinaus, wenn sie sich allgemein in der Form der Reklamation von Ansprüchen ausflaggen. Soziologisch formuliert, kann es sogar offen bleiben, ob es Interessen tatsächlich gibt; es genügt, davon auszugehen, dass Kommunikation auf sie referiert.

Interessen sind in dieser Lesart funktionale Grenzmarkierungen der Politik: die politische Markierung von Interessen ist vergleichbar mit der kommunikativen Zuschreibung von bewussten Intentionen, sie fungiert als produktive Unterbrechung der eigenen Beobachtungen durch bestimmte Unterscheidungen.

Die »Kontraktualisierung des Politischen ${ }^{21}$ meint in diesem Sinne: das Politische soll von seinen Schattenseiten und Irrationalitäten befreit und darin rationalisiert werden. Entfesselte Interessenkämpfe und individuelles Machtstreben gehen in politischer Ordnung auf.

Es ist nun das eine, diesem institutionalistischen oder kontraktualistischen Blick auf das Politische die Diagnose einer Verflüchtigung oder Diffundierung des Politischen beiseite zu stellen. Eine solche Perspektive beobachtet dann, dass alternative Gestaltungsund Steuerungsformen mehr Gewicht erhalten, und dass das Politische sich aus den dafür vorgesehenen Institutionen verflüchtigt oder auch ausweitet, so dass es zu einer Art Entstaatlichung der Politik kommt.

Die Unterscheidung politisch/subpolitisch ist hier allerdings nicht entscheidend, auch wenn sie sinnvolle Analysen nahe legt. Die Unterscheidung politischer /vorpolitischer Interessen legt hiergegen einen Zugang nahe, der analytisch schwer dingfest gemacht werden kann und auch in normativer Hinsicht nicht einfach der Prämierung ziviligesellschaftlicher Interessen genügt.

Vorpolitische und politische Interessen bilden ein wechselseitiges Durchdringungsverhältnis. Dekomponierbare, rationalisierbare Interessen treffen auf existentiale. Hier wird es möglich, die Arendt sche Differenz von politischen/unpolitischen Wesen zu erweitern. Für Arendt war Macht gleichbedeutend mit der Handlungsfähigkeit und Handlungsbereitschaft einer sozialen Gruppe und dabei das Moment der Zustimmung das entscheidende. Die Macht einer Gruppe findet ihre Grenze in der Macht anderer Gruppen, insofern diese nicht in Sicht sind, kann sich die Macht der einen Gruppe stabilisieren, kann auf absehbare Zeit "große Reiche und zahllose Menschen beherrschen «. ${ }^{22}$ Macht wird von Arendt dabei in einem Ausschlussverhältnis zur Gewalt behandelt. Gewalt kann Macht nur zerstören, sie kann sich aber nicht an ihre Stelle setzen. Macht verschwindet vielmehr im Zuge des Zusammenhalts der Gruppe. Nicht die Frage des Machterhalts oder der Ausübung der Gewalt steht im Mittelpunkt des Arendt`schen Interesses, sondern einzig und allein das Moment der Entstehung und des Aufblühens der Macht: »Mit realisierter Macht haben wir es immer dann zu tun, wenn Worte und Taten untrennbar miteinander verflochten erscheinen, wo also Worte nicht leer und Ta-

21 Christian Lahusen, Die Kontraktualisierung des Politischen, In: Armin Nassehi/Markus Schroer, Der Begriff des Politischen, Baden Baden 2003, S. 101-117.

22 Arendt, Elemente und Ursprünge totaler Herrschaft, a.a.O., S. 194.

ZfP 56. Jg. 3/2009 
ten nicht gewalttätig stumm sind, wo Worte nicht missbraucht werden, um Absichten $\mathrm{zu}$ verschleiern, sondern gesprochen werden, um Wirklichkeiten zu enthüllen, und wo Taten nicht missbraucht werden, um zu vergewaltigen und zu zerstören, sondern um neue Bezüge zu etablieren und zu festigen, und damit neue Realitäten zu schaffen.«23

Hier liegt offensichtlich auch der tiefere Grund für Arendt, um so rigide zwischen politisch/unpolitisch zu unterscheiden: Macht zeigt sich entweder als ein idealer sozialer Prozess, in dem sich keine falschen Interessen gewaltförmig zusammenfügen; der Begriff des Politischen wird hier radikal von dem der Gewalt getrennt. Die Hobbesianische Traditionslinie wird dort durchbrochen, wo sich der - wenn auch flüchtige - Augenblick des Zusammenhandelns offenbart und die Kluft zwischen Machtüberlegenen und Machtunterlegenen eingeebnet wird. Das Ausgeschlossene dessen aber findet sich in der Masse der Unpolitischen, der Gewalttätigen, in der Ansammlung falscher Machtinteressen.

Die Unterscheidung von politischen und vorpolitischen Interessen kann dieses $\mathrm{Di}$ lemma nicht aus der Welt schaffen, aber es kann aus der Verlegenheit helfen, diese Unterscheidung mit politischen/unpolitischen Lebewesen ineins setzen zu müssen. Der Handlungsbegriff als solcher wird phänomenologisch aufgefächert, als er nicht mehr auf politische Interessen hin alleine fixiert wird.

Der Begriff des Vorpolitischen, bzw. des vorpolitischen Interesses lässt sich nun vorsichtig konkretisieren: er umschreibt - wenn man so will - das Unbestimmbare, vielleicht auch Unbezähmbare und nicht restlos Logofizierbare konkreter Gemeinschaften, in dessen Rahmen verschiedene Dimensionen miteinander in Anschlag gebracht werden:

Arendt sah in der menschlichen Fähigkeit zum Handeln einerseits die Idee revolutionärer Weltveränderung angelegt; im Handeln enthüllte sich für sie die Sache der Freiheit, die das eigentliche Wesen der Politik zum Ausdruck bringe. Die ursprungsphilosophische Semantik und das Paradigma eines politischen Neuanfangs blieb für sie aber in einem gewissen Grade unvorhersehbar, unberechenbar. Freiheit war für sie performatives Freisein, das einen Abgrund eröffnet und dem Fluch der Kontingenz verbunden bleibt. Freiheit war für sie mit dem Einbruch des jäh und schlechthin Unberechenbaren verbunden, gegenüber dem man sich zu keiner Zeit und an keinem Ort wappnen kann, das den beruhigenden Ordnungsrahmen jedes verfügungsrationalistischen Differenzschemas sprengt. $^{24}$

Es ist die Freiheit eigeninitiativen Handelns, die als vorpolitische Fähigkeit des Menschen die Entstehung konkreter politische Institutionen ermöglicht. Die Ambivalenz der politischen Anthropologie findet sich in der Differenz zwischen dem politischen und dem vorpolitischen Begriff des Handelns, der zu normativen Engpässen führt: denn er blendet das Unpolitische aus, oder einfacher:

unpolitische Menschen - für die man dann in der modernen ausdifferenzierten Gesellschaft eine Übersetzung finden müsste - haben an sich keinen Anspruch auf bürgerliche Gleichheit, sie stellen vielmehr eine latente Gefahr für die Existenz der Polis dar,

23 A.a.O., S. 195.

24 Arendt, Vita activa, a.a.O., S. 166. 
während allen Menschen gleichzeitig qua Natalität eine egalitär verteilte Freiheit zugesprochen wird.

Es ist das alte Problem des ungelösten Ausgangszustands zwischen vorpolitischer Latenz und konkreter politischer Performanz: Man ist gezwungen, Ausgangssituationen zu konstruieren, die durch universalistische normative Prinzipien gekennzeichnet sind: etwa des menschenrechtlichen Grundverhältnisses der Anerkennungswechselseitigkeit freier und gleicher Personen. Entlang der Frage der konkreten politischen Gestaltung wird man aber stets auf die ursprungsphilosophische Ausgangssituation zurückverwiesen. Das kategoriale Element der Handlungsfreiheit weist zwar den Weg aus dem Naturzustand in Richtung eines wechselseitigen Freiheitsverzichts. Dieses setzt aber eine rechts- und moraluniversalistische Beurteilungsperspektive immer schon voraus, die auf Wechselseitigkeit, reziproker Anerkennung und menschenrechtlich abgesicherten Grundverhältnissen aufzubauen, resp. konkret zu entfalten wäre: Die universalistischen Konstituentien dieser Ausgangssituation können die Gelingensbedingungen rechtfertigungslogisch nicht einholen, denn sie führen in den Bereich der transzendentalen Voraussetzungen und decken dabei diejenigen Bedingungen auf, die von jedem immer schon als erfüllt und gesichert betrachtet werden müssen, um überhaupt Interessen realisieren zu können.

Wenn sich die Individuen aber erst dann zu einem Freiheitsverzicht bereit finden, wenn sie sich in einer Situation des relativen Gleichgewichts befinden, dann scheint dies für die rechtsmoralische Verfassung der Weltgesellschaft auf prekäre Konsequenzen hinauszulaufen, da sich für die Mehrheit der von sozialräumlicher Exklusion bedrohten Individuen die Frage von vornherein nicht stellt. ${ }^{25}$

Hiergegen ist es aber sinnvoll davon auszugehen, dass politische Interessen von vorpolitischen Interessen flankiert werden; diese bilden zwar den Hauptgegenstand sozialwissenschaftlicher Analysen, während jene aber zumindestens das Politische mit bedingen, wenn auch nicht determinieren können. Das Begründungsprogramm des politischen Liberalismus ist damit nicht $\mathrm{zu}$ verlassen, aber seine politische Basismelodie ist mit denjenigen vorpolitischen Obertönen abzugleichen, die sich an den »Modernitätskonflikten in der Weltgesellschaft « entzünden. ${ }^{26}$

Die allgemein konsensfähige und im normativen Sinne hoch plausible Antwort lautet: eine demokratische Verfassung setzt keine Homogenität, sondern nur das Erlernen einer demokratischen Kultur voraus; entlang rechtsstaatlicher Garantien, die den Einfluss aktueller Minderheiten auf die Willensbildung von Staat und Gesellschaft aufrecht erhalten und insofern Freiräume schaffen, in denen Einzelne ihre Vorstellungen des guten und richtigen Lebens im Rahmen der Legalität artikulieren können. Ein solchermaßen ermöglichter minimalistischer Zusammenhalt benötigt keine vorpolitischen Homogenitätsvoraussetzungen, die - monistisch zugespitzt - eher im Gegensatz zu den Gelingensbedingungen demokratischer Gesellschaften stehen.

25 Wolfgang Kersting, Recht, Gerechtigkeit und demokratische Tugend, Frankfurt a. M. 1997.

26 Thomas Bonacker, Modernitätskonflikte in der Weltgesellschaft. Zur kulturellen Konstruktion globaler Konflikte. In: Soziale Welt (57) 2006, S. 47-63. 
Sinnvoll erscheint in analytisch-konflikttheoretischer Sicht aber ein theoretisches Arrangement, das von einem Bedingungsverhältnis politischer und vorpolitischer Interessen ausgeht: sind diese relativ gut gedeckt, fallen jene weniger ins Gewicht; politisch wenig integrierte, von kulturellen, ethno-politischen und substaatlichen Konflikten geprägte Gesellschaften hingegen spüren die Wucht vorpolitischer Interessen, die dann zwar auf die Artikulation des Unrechts zielen, sich aber ebenso einer fatalen negatorischen Moral verschreiben können. Anstatt also demokratische Kultur als etwas zu verstehen, das von außen vorgeschrieben oder gar importiert werden kann, mag eine nüchterne Relationierung von politischen und vorpolitischen Interessenkonstellationen einen reflexiven Vorteil bringen. Man umgeht zumindestens die Schwierigkeiten einer Ausgangszustandsparadoxie, von der Philosophien der Weltbürgergesellschaft träumen; man umgeht ferner eine staatszentrierte Wirklichkeitssicht, die das Vorpolitische vollkommen in einem asketischen Egalitarismus aufgehen sieht; bescheidener erscheint der Anspruch einer weltpolitischen Verfassung, in der ungebundene vorpolitische Quellen nicht erstickt oder vollkommen rationalisiert, sondern mit den gegebenen Mitteln des Rechts umhegt werden, was selbstredend auf bekannte politische Desiderate verweist. Kritisch wird die Unterscheidung politisch/vorpolitisch in diesem Zusammenhang daher, insofern sie die Balance zwischen den gängigen politischen Interessenartikulationen und dem damit verknüpften Realismus einerseits, der soziologischen Einsicht in die weltweit vorenthaltene Anerkennungsverhältnisse andererseits aufrecht erhält; sie darüber hinaus aber die Bruchstellen und Risse im Interessenhaushalt der Moderne überhaupt erst artikulierbar macht: zugespitzt im Ausgangszustandsproblem einer weltbürgerlichen Verfassung, bei der homogene und verrechenbare Interessen immer schon vorausgesetzt werden. Über die einschlägigen rechtstheoretischen Diskurse hinaus greift der Begriff des Vorpolitischen das Faktum uneingelöster Interessen heraus, ohne sie auf das Niveau von konsentierbaren Geltungsansprüchen zu heben.

Gängige Konfliktschemata verlieren damit zwar nicht ihre Gültigkeit, aber sie werden entlang von Neotribalismen, fundamentalistischen Gruppendeutungen und den Bedingungen des Massenhandelns unter prekären herrschaftlichen Bedingungen, also entlang der »disseminalen « Zerstreuung von Interessenkonstellationen kontingent. In den Mittelpunkt eines solchermaßen verstandenen Konfliktverständnisses rückt damit eine Form der Prozessualität, die einen konstitutiven Bruch zwischen Strategien der Einschließung und Taktiken der Öffnung erkennt und damit nicht ein pyramidales, sondern nicht-lineares Arrangement sozialer Kämpfe zugrunde legt. Diese sind eben in der Nichtberechenbarkeit von Kämpfen um Anerkennung zu verorten: Das Ringen um Macht ist nicht auf Machtlosigkeit alleine und die Gewalt nicht allein auf vorenthaltene Anerkennung alleine zu reduzieren, sondern sie sind gewissermaßen subjektpolitische Interpretationen von Prozessen, die im Vorpolitischen in Gang gebracht werden. 


\section{Zusammenfassung}

Der vorliegende Aufsatz möchte versuchen, den Begriff des vorpolitischen Interesses als eine eigenständige Dimension des Politischen einzuführen und ihn als Alternative zur Differenz von vorpolitischem Fundamentalismus und politischer Moderne zu beschreiben.

Im einzelnen soll der Begriff des vorpolitischen Interesses entlang klassischer politischer Entwürfe konturiert werden; zunächst in Opposition zum Horizont einer vorpolitischen Homogenität bei Heidegger und Schmitt (1); anschließend in einer ausführlichen Auseinandersetzung mit der politischen Theorie Hanna Arendt's (2). Deren politischer Freiheitsbegriff ist einerseits maßgeblich für die Explikation des vorpolitischen Sinnhorizonts; zugleich eröffnet er offene Flanken, von denen hier vor allem die rigide Unterscheidung von politischen und vorpolitischen Interessen $\mathrm{zu}$ nennen ist (3). Das Schema von Fundamentalismus und Moderne bringt in diesem Zusammenhang die Schwierigkeit der politischen Theorie zum Ausdruck, vorpolitische Interessen als eigenständige Dimension des Politischen anzuerkennen.

\section{Abstract}

The article tries to introduce the idea of »prepolitical interests « as an autonomous category of Political Theory. It refers on the difference between fundamental and traditional political interests, described along traditional political concepts, (1.) in opposition to the understanding of the prepolitical dimension (Heidegger, Schmitt), further along Hannah Arendt's political Theory (2.). Arendt's political understanding of freedom helps to explain the prepolitical dimension, though it refers to an important contradiction (3). The pattern fundamentalism/modern society has so far no real expression for political interests, which lie before or beyond usual political interests.

Christian Wevelsiep, Political and pre-political interests - Political Theory after Heidegger and Arendt

ZfP 56. Jg. 3/2009 